\title{
Pneumocystis pneumonia in a patient with lupus nephritis
}

\author{
${ }^{1}$ Department of Internal Medicine, Sind Institute of Urology and Transplantation, Karachi, Pakistan \\ ${ }^{2}$ Dow Medical College, Civil Hospital Karachi, Pakistan \\ ${ }^{3}$ Department of Histopathology, Sind Institute of Urology and Transplantation, Karachi, Pakistan
}

Zain Majid $^{{ }^{*}}$, Afshan Hussain ${ }^{2}$, Hafsa Jawaid ${ }^{2}$, Shoaib Ahmed ${ }^{1}$, Saima Ahmed ${ }^{1}$, Mohammad Mubarak ${ }^{3}$

\section{A R T I C L E IN F O}

Article Type:

Case Report

\section{Article History:}

Received: 13 September 2017

Accepted: 4 December 2017

ePublished: 11 December 2017

\section{Keywords:}

Lupus nephritis

Pneumocystis pneumonia

Bronchoalveolar lavage

Sulfamethoxazole and trimethoprim

Immunosuppressive therapy

\begin{abstract}
A B S T R A C T
Patients with lupus nephritis on immunosuppressive therapy are at an increased risk of opportunistic infections. Pneumocystis pneumonia infection though rare, does occur in such patients and is usually missed on commonly conducted investigations. Hence physicians must suspect it when clinical signs and symptoms do not improve in these patients despite being on treatment. being a diagnosed case of lupus nephritis (renal biopsy proven; immunofluorescence microscopy (IMF) showed full house positivity while serum serology revealing decreased levels of both serum $\mathrm{C} 3$ and $\mathrm{C} 4$, a strong positive ANA and rheumatoid factor). She had previously received three doses of cyclophosphamide. She was referred to us with the complains of pedal edema, fever and cough. Her examination revealed bilateral lung crepitations. Chest X-ray showed well defined shadows adjacent to the lung hilum with air bronchogram. In her bronchoalveolar lavage, a few pseudohyphae along with a positive test for Pneumocystis jiroveci pneumonia (PCP) was detected. Hence, the patient was treated for PCP pneumonia with sulfamethoxazole and trimethoprim. After completion of the course her condition had improved significantly and she was later on discharged.
\end{abstract}

Implication for health policy/practice/research/medical education:

Patients with lupus nephritis on immunosuppressive therapy are at an increased risk of opportunistic infections. Pneumocystis pneumonia infection though rare, does occur in such patients and is usually missed on commonly conducted investigations. Hence physicians must suspect it when clinical signs and symptoms do not improve in these patients despite being on treatment. Please cite this paper as: Majid Z, Hussain A, Jawaid H, Ahmed S, Ahmed S, Mubarak M. Pneumocystis pneumonia in a patient with lupus nephritis. J Nephropharmacol. 2017;7(1):40-42. DOI: 10.15171/npj.2018.09.

\section{Introduction}

Patients with lupus nephritis on immunosuppressive therapy are at an increased risk of opportunistic infections. Pneumocystis pneumonia infection though rare, does occur in such patients and is usually missed on commonly conducted investigations. Hence physicians must suspect it when clinical signs and symptoms do not improve in these patients despite being on treatment.

\section{Case Presentation}

A 20 year old female diagnosed case of lupus nephritis class IV and V, based upon her renal biopsy. Renal biopsy proven. Her immunofluorescence microscopy (IMF) showed fullhouse positivity (Figure 1) while serum serology revealed decreased levels of both serum C3 and C4, a strong positive ANA and a positive rheumatoid factor. She had previously received three doses of immunosuppressive therapy with cyclophosphamide. She presented this time with pedal edema along with undocumented fever on and off, cough with yellowish sputum since four days. She was currently on immunosuppressive therapy (prednisolone $15 \mathrm{mg} / \mathrm{d}$ ) and for her current complaints she was admitted and started on IV broad spectrum antibiotics (Tazobactam).

On examination she was vitally stable, having bilateral crepitation on chest auscultation, while rest of her examination was unremarkable. Her initial laboratory reports showed a low hemoglobin of $9.2 \mathrm{~g} / \mathrm{dL}$ along with a raised serum creatinine $2.3 \mathrm{mg} / \mathrm{dL}$ (Her previous serum creatinine was $0.7 \mathrm{mg} / \mathrm{dL} 2$ months ago). Rest of her laboratory results were normal.

For these complains her chest X-ray showed well defined shadows adjacent to her right and left lung hilum with air bronchogram, more developed on the right side (Figure 2). Since she was producing sputum, her sputum sample was 


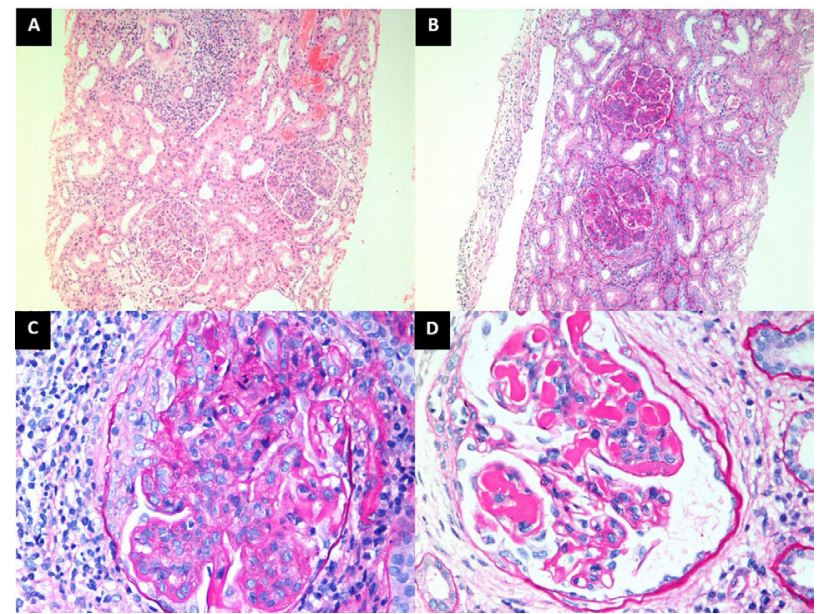

Figure 1. Histopathological features on renal biopsy. A. Lowpower view showing grossly abnormal glomeruli and patchy dense interstitial inflammation. (H\&E, ×100). B. Another area of biopsy showing markedly hypercellular glomeruli with prominent lobulation (PAS, $\times 100$ ). C. High-power view showing mesangiocapillary pattern of morphology with a small crescent at 9'O clock position. (PAS, $\times 400)$. D. This glomerulus shows numerous hyaline thrombi and wire loop lesions, characteristically found in lupus nephritis (PAS, $\times 400$ ).

sent for detailed report, culture and sensitivity and acidfast bacilli (AFB) smear but all tests were negative.

Her viral markers were also negative. Her blood and urine cultures showed no growth while her Urine D/R was bland.

Since her chest symptoms did not improve and she was not maintaining her saturation, she was kept on intermittent oxygen support of 2 L. Patient's antibiotics were escalated to imipenem. Later on her bronchoalveolar lavage (BAL) pseudohyphae along with testing positive for pneumocystis pneumonia (PCP). Then, the patient was managed for PCP pneumonia and started on sulfamethoxazole and trimethoprim. After completion of the course her condition had improved significantly and she was later on discharged.

\section{Discussion}

Systemic lupus erythematosus (SLE) is an autoimmune disease characterized by multisystem involvement and the production of various autoantibodies (1). SLE may involve any organ, the most common ones being the skin, joints, kidneys, heart, lungs and CNS (2). Involvement of kidneys named lupus nephritis (LN) affects $20 \%$ to $75 \%$ of patients in the first 10 years of illness (3).

Development and progression of $\mathrm{LN}$ is regarded as a multistep inflammatory process which is incited by anti-DNA and anti-nucleosome antibodies, resulting in a self-maintaining inflammatory loop with spreading of glomerular inflammation. Pro-inflammatory antibodies such as $\mathrm{Clq}$ are involved (4). Early manifestations of LN include hematuria and proteinuria revealed by urinalysis (5). The diagnosis is however confirmed

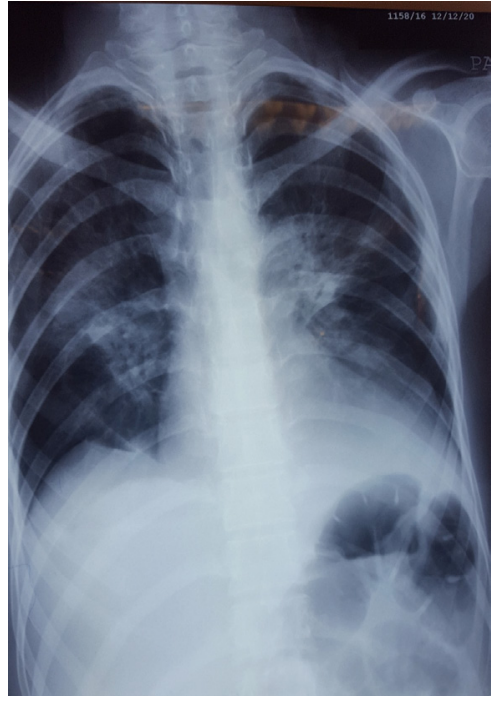

Figure 2. Chest X-ray, PA view showing well defined shadows adjacent to her right and left lung hilum with air bronchogram, more developed on the right side

by biopsy results where the disease is staged as per different histopathological presentations (5). Intravenous cyclophosphamide combined with corticosteroids is the first line therapy for lupus nephritis (6). The resultant immunosuppression increases the risk of opportunistic infections.

However unlike most opportunistic infections, pneumocystis pneumonia (PCP) has a low prevalence of $0.45 \%$ in immunocompromised SLE patients (7). Lower lymphocyte count right before the onset of PCP and the higher dose of steroid therapy along with depressed CD4+ counts are considered possible risk factors for the development of PCP infection in SLE patients (8). This elevated risk is attributed to an altered immune response in the patients and adverse reactions to immunosuppressive therapy.

PCP typically presents with fever, non-productive cough, shortness of breath, weight loss and night sweats. Clinical diagnosis of PCP is made by using PCR of sputum, serum beta-D glucan test, lung biopsy and bronchoalveolar lavage $(9,10)$. Non-invasive and reliable serum indicator of PCP and serum beta-D-glucan hold significant importance in the definitive diagnosis, especially when severe respiratory distress impedes bronchoalveolar lavage (BAL) (11).

PCP infection rarely contributes to a high mortality rate $(60 \%)$ and can be a life threatening condition (12). According to two studies, all SLE patients who developed PCP, correlated with poor clinical outcome and massive kidney involvement $(12,13)$. Severity and treatment of PCP may be different in different ethnicities. In the Asian population it may be more progressive and requires more effective treatment (14). Trimethoprim-sulfamethoxazole is reported to be an important constituent in the treatment regimen of PCP where the antibiotic shows a favorable 
response (9).

\section{Conclusion}

Our study highlights the importance of diagnosis and treatment of all infections, in particular, in patients with lupus nephritis who are currently on immunosuppressive therapy. Physicians catering to such patients need to keep in mind common infections as well as rare entities like PCP, since the spectrum of disease infecting these patients can be very vast.

\section{Conflicts of interest}

There were no points of conflicts.

\section{Authors' contribution}

ZM managed the patient and wrote the manuscript. ShA managed the patient. SaA managed the patient. $\mathrm{AH}$ and HJ wrote the manuscript. MM was responsible for the histopathological diagnosis and images. All authors read and signed the final paper.

\section{Ethical considerations}

Ethical issues (including plagiarism, data fabrication, double publication) have been completely observed by the authors. Written informed consent was obtained from the patient for publication this case report.

\section{Funding/Support}

None.

\section{References}

1. Anders HJ, Fogo AB. Immunopathology of lupus nephritis. Semin Immunopathol. 2014;36:443-59. doi: 10.1007/s00281-013-0413-5.

2. Silva CA, Aikawa NE, Pereira RM, Campos LM. Management considerations for childhood-onset systemic lupus erythematosus patients and implications to therapy. Expert Rev Clin Immunol. 2015;15:1-13. doi:10.1586/174 4666X.2016.1123621.

3. Cervera R, Khamashta MA, Hughes G. The Euro-lupus project: epidemiology of systemic lupus erythematosus in
Europe. Lupus 2009; 18:869-874.

4. Gatto M, Iaccarino L, Ghirardello A, Punzi L, Doria A Clinical and pathologic considerations of the qualitative and quantitative aspects of lupus nephritogenic autoantibodies: a comprehensive review. J Autoimmun. 2016;69:1-11. doi: 10.1016/j.jaut.2016.02.003.

5. Mohan C, Putterman C. Genetics and pathogenesis of systemic lupus erythematosus and lupus nephritis. Nat Rev Nephrol. 2015;11:329-41. doi: 10.1038/nrneph.2015.33.

6. Palmer SC, Tunnicliffe DJ, Singh-Grewal D, Mavridis D, Tonelli M, Johnson DW et al. Induction and maintenance immunosuppression treatment of proliferative lupus nephritis: A network meta-analysis of randomized trials. Am J Kidney Dis. 2017;70:324-336. doi: 10.1053/j. ajkd.2016.12.008.

7. Kapoor TM, Mahadeshwar P, Nguhen S, Li J, Kapoor S, Bathon J, et al. Low prevalence of pneumocystis pneumonia in hospitalized patients with systemic lupus erythematosus: review of a clinical data warehouse. Lupus. 2017;26:14731482. doi: $10.1177 / 0961203317703494$

8. Lertnawapan R, Totemchokchyakarn K, Nantiruj K, Janwityanujit S. Risk factors of Pneumocystis jeroveci pneumonia in patients with systemic lupus erythematosus. Rheumatol Int. 2009;29:491-6. doi: 10.1007/s00296-0080721-6.

9. Wainstein E, Neira O, Guzman L. Lupus erythematosus disseminatus and Pneumocystis carinii pneumonia. Rev Med Chil. 1993;121:1422-5.

10. Su GX, Wu FQ, Wang F, Zhou ZX, Huang XL, Lu J. Rituximab therapy for severe pediatric systemic lupus erythematosus. Zhonghua Er Ke Za Zhi 2012; 50:697-704.

11. Tasaka S1, Hasegawa N, Kobayashi S, Yamada W, Nishimura T, Takeuchi T, et al. Serum indicators for the diagnosis of pneumocystis pneumonia. Chest 2007;131:1173-80. doi: 10.1378/chest.06-1467..0

12. Weng CT, Liu MF, Weng MY, Lee NY, Wang MC, Lin WC, et al. Pneumocystis jirovecii pneumonia in systemic lupus erythematosus from southern Taiwan. J Clin Rheumatol 2013;19:252-8. doi: 10.1097/RHU.0b013e31829d5017.

13. Tang XY, Li J, Dong F, Song HM. Clinical characteristics of pneumocystis pneumonia in children with lupus erythematosus. Zhonghua Er Ke Za Zhi. 2013;51:920-4.

14. Tesar V, Hruskova Z. lupus nephritis; a different disease in european patients? Kidney Dis (Basel). 2015;1:110-8.

Copyright $\odot 2018$ The Author(s); Published by Society of Diabetic Nephropathy Prevention. This is an open-access article distributed under the terms of the Creative Commons Attribution License (http://creativecommons.org/licenses/by/4.0), which permits unrestricted use, distribution, and reproduction in any medium, provided the original work is properly cited. 